\title{
Exploring the nature of the unidentified very-high-energy gamma-ray source HESS J1507-622
}

\author{
W. Domainko ${ }^{1}$ and S. Ohm ${ }^{2,3}$ \\ 1 Max-Planck-Institut für Kernphysik, PO Box 103980, 69029 Heidelberg, Germany \\ e-mail: wilfried.domainko@mpi-hd.mpg.de \\ 2 Department of Physics \& Astronomy, University of Leicester, UK \\ 3 School of Physics \& Astronomy, University of Leeds, Leeds LS2 9JT, UK
}

Received 17 November 2011 / Accepted 8 August 2012

\begin{abstract}
Context. Several extended sources of very-high-energy (VHE; $E>100 \mathrm{GeV}$ ) gamma rays have been found that lack counterparts belonging to an established class of VHE gamma-ray emitters.

Aims. The nature of the first unidentified VHE gamma-ray source with significant angular offset from the Galactic plane of $3.5^{\circ}$, HESS J1507-622, is explored.

Methods. Fermi-LAT data in the high-energy (HE, $100 \mathrm{MeV}<E<100 \mathrm{GeV}$ ) gamma-ray range collected over 34 month are used to describe the spectral energy distribution (SED) of the source. Additionally, implications of the off-plane location of the source for a leptonic and hadronic gamma-ray emission model are investigated.

Results. HESS J1507-622 is detected in the Fermi energy range and its spectrum is best described by a power law in energy with $\Gamma=1.7 \pm 0.1_{\text {stat }} \pm 0.2_{\text {sys }}$ and integral flux between $(0.3-300) \mathrm{GeV}$ of $F=\left(2.0 \pm 0.5_{\text {stat }} \pm 1.0_{\text {sys }}\right) \times 10^{-9} \mathrm{~cm}^{-2} \mathrm{~s}^{-1}$. The SED constructed from the Fermi and H.E.S.S. data for this source does not support a smooth power-law continuation from the VHE to the HE gamma-ray range. With the available data it is not possible to discriminate between a hadronic and a leptonic scenario for HESS J1507-622. The location and compactness of the source indicate a considerable physical offset from the Galactic plane for this object. In case of a multiple-kpc distance, this challenges a pulsar wind nebula (PWN) origin for HESS J1507-622 since the time of travel for a pulsar born in the Galactic disk to reach such a location would exceed the inverse Compton (IC) cooling time of electrons that are energetic enough to produce VHE gamma-rays. However, an origin of this gamma-ray source connected to a pulsar that was born off the Galactic plane in the explosion of a hypervelocity star cannot be excluded.

Conclusions. The nature of HESS J1507-622 is still unknown to date, and a PWN scenario cannot be ruled out in general. On the contrary HESS J1507-622 could be the first discovered representative of a population of spatially extended VHE gamma-ray emitters with HE gamma-ray counterpart that are located at considerable offsets from the Galactic plane. Future surveys in the VHE gamma-ray range are necessary to probe the presence or absence of such a source population.
\end{abstract}

Key words. gamma rays: ISM - pulsars: general - ISM: supernova remnants - radiation mechanisms: non-thermal

\section{Introduction}

The inner Galaxy has been surveyed by the H.E.S.S. telescope array in very-high-energy (VHE, $E>100 \mathrm{GeV}$ ) gamma rays and during this survey several sources of such radiation have been found which lack obvious counterparts in other wavelength (Aharonian et al. 2008). VHE gamma rays are in many cases expected to be produced either by inverse Compton (IC) scattering of low energy photons from e.g. stellar radiation fields or the cosmic microwave background radiation by VHE electrons (leptonic channel) or by $\pi^{0}$ decay following inelastic collisions between hadronic cosmic rays and target protons and nuclei from the ambient medium (hadronic channel, see Hinton \& Hofmann 2009 , for a review). Both channels could in principle explain the characteristics of the significant population of unidentified VHE gamma-ray sources which lack obvious counterparts in other wavelength. Evolved pulsar wind nebulae (PWN) as leptonic sources (de Jager 2008; Mattana et al. 2009) or old supernova remnants (SNRs) as hadronic sources (Yamazaki et al. 2006) have been proposed to be viable object candidates. Both scenarios have in common that they assume a rather large object age of $>10^{4}$ years. Indeed, as in the case of the formerly unidentified sources HESS J1616-508 (Aharonian et al. 2005) and HESS J1731-347 (Aharonian et al. 2008), both could be associated to an evolved PWN (Landi et al. 2007) and an old SNR system (Tian et al. 2008; Abramowski et al. 2011a), respectively. However, the gamma-ray production processes in a large fraction of unidentified VHE gamma-ray sources are still not known to date.

One possibility to distinguish between a leptonic and a hadronic scenario for a specific source is to utilise observations in the to the VHE gamma-ray range adjacent band of highenergy (HE, $100 \mathrm{MeV}<E<100 \mathrm{GeV}$ ) gamma rays (see e.g. Tam et al. 2010; Abdo et al. 2011). Torres et al. (2011) demonstrated the potential of this approach by using data obtained in observations with the Large Area Telescope (LAT) aboard the Fermi satellite to investigate the nature of the unidentified VHE gamma-ray source HESS J1858+020.

In the HE gamma-ray band, observations of objects located close to the Galactic plane are severely affected by diffuse gamma-ray emission originating from interactions of $\mathrm{HE}$ electrons and hadrons with interstellar radiation fields and interstellar matter. Thus strong, unidentified VHE gamma-ray sources with an offset from the Galactic plane are ideal for 
such an investigation of the radiation mechanism. Recently, such an object with an angular offset from the Galactic plane has indeed been found: HESS J1507-622 (Acero et al. 2011). This source is detected with an integral flux above $1 \mathrm{TeV}$ of $F_{\gamma}(>1 \mathrm{TeV})=\left(1.5 \pm 0.4_{\text {stat }} \pm 0.3_{\text {sys }}\right) \times 10^{-12} \mathrm{~cm}^{-2} \mathrm{~s}^{-1}$ and a power-law spectrum $\mathrm{d} N / \mathrm{d} E=k(E / 1 \mathrm{TeV})^{-\Gamma}$ with spectral in$\operatorname{dex} \Gamma=2.24 \pm 0.16_{\text {stat }} \pm 0.20_{\text {sys }}$ and a flux normalization of $k=(1.8 \pm 0.4) \times 10^{-12} \mathrm{TeV}^{-1} \mathrm{~cm}^{-2} \mathrm{~s}^{-1}$. For this particular object, Domainko (2011) showed that it is difficult to explain the gamma-ray emission to originate from an old SNR. An attempt was made to explain HESS J1507-622 in the framework of an ancient PWNe scenario (Tibolla et al. 2011). So far no pulsar could be identified in the vicinity of the H.E.S.S. source.

Recently a GeV counterpart to HESS J1507-622, namely 2FGL J1507.0-6223, has been reported in the Fermi 2-year catalogue (Nolan et al. 2012). In this paper an extended 34 month Fermi-LAT data set is used to investigate gamma-ray emission models of HESS J1507-622 using the differential energy spectrum from $\mathrm{GeV}$ to $\mathrm{TeV}$ energies. Additionally, radio and $\mathrm{X}$-ray measurements from the literature (Acero et al. 2011; Murphy et al. 2007) are adopted to further constrain the spectral energy distribution (SED). Furthermore, its location offset from the Galactic plane is used to set constraints on the nature of the source.

\section{Fermi-LAT analysis}

The Fermi-LAT is a pair-conversion telescope operating in the $20 \mathrm{MeV}$ to $300 \mathrm{GeV}$ energy range. The direction of incident photons is reconstructed by means of a tracker. A calorimeter is used to measure the energy of the incident particle, and an anticoincidence system efficiently suppressed the charged-particle background. The large field-of-view of the instrument of $\sim 2.4 \mathrm{sr}$ allows to cover the whole sky every two orbits. The LAT provides an angular resolution of $<1^{\circ}$ at $1 \mathrm{GeV}$ and $<0.2$ at $10 \mathrm{GeV}$. A full mission- and instrument-related description can be found in Atwood et al. (2009).

Based on 24 months of data, the HE gamma-ray source 2FGL J1507.0-6223 has been reported to emit gamma-ray emission in the HE regime (Nolan et al. 2012). Within the $1 \sigma$ positional uncertainty, 2FGL J1507.0-6223 is consistent with the best fit position of HESS J1507-622 (angular separation of 3.6') which makes a physical association of the Fermi-LAT and the H.E.S.S. source very likely. The best fit model to the SED of 2FGL J1507.0-622 reported in Nolan et al. (2012) is a power law in energy $\left(\mathrm{d} N / \mathrm{d} E \propto E^{-\Gamma}\right)$ with differential index $\Gamma=1.9 \pm 0.2$ and an integral flux between $(1-100) \mathrm{GeV}$ of $(1.0 \pm 0.3) \times$ $10^{-9} \mathrm{~cm}^{-2} \mathrm{~s}^{-1}$. Note that in the catalogue significant emission is reported only in the (10-100) GeV band and flux upper limits are given at lower energies.

The analysed data set used in this work has been recorded in observations over 34 months of operation from the commissioning of Fermi in August 2008 until May 2011. Data analysis has been performed using events with reconstructed energies between $300 \mathrm{MeV}$ and $300 \mathrm{GeV}$, utilising the Fermi Science Tools (FST) package, version v9r23p $1^{1}$. Events have been selected according to and analysed with the P7Source_V6 instrument response functions (IRFs).

The best fit position of HESS J1507-622 has been used to define a Region-of-Interest (RoI) of $10^{\circ}$ from which events have been included in the maximum likelihood analysis. All sources

\footnotetext{
1 http://fermi.gsfc.nasa.gov/ssc/data/analysis/ documentation/Cicerone/
}

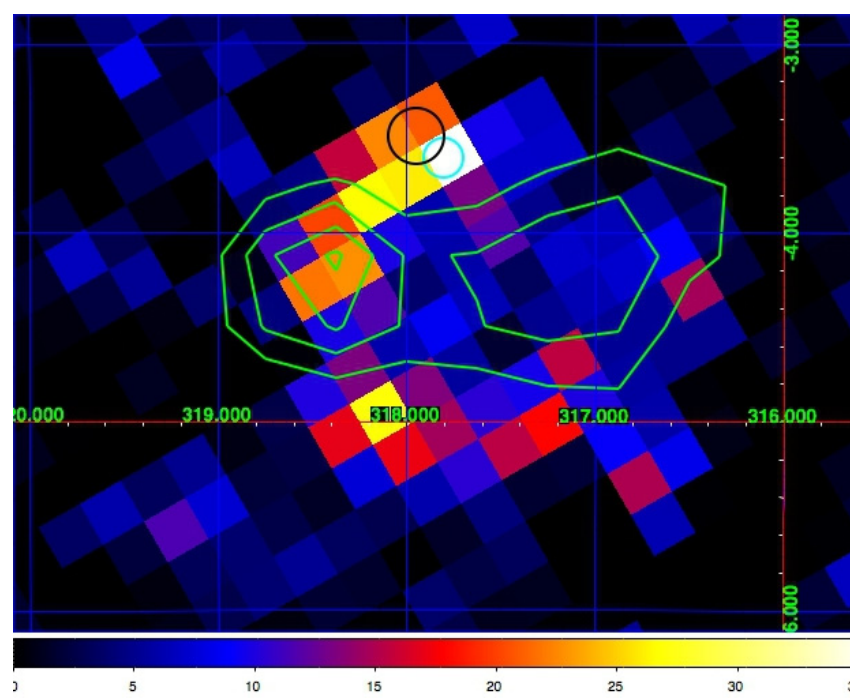

Fig. 1. Test statistics map for photon energies $10 \leq E \leq 300 \mathrm{GeV}$. The cyan circle denotes the error circle of the best fit position of the LAT source in this energy band and the black circle indicates the intrinsic width of the H.E.S.S. source. Overlaid in green are CO contours (Dame et al. 2001) of the foreground molecular cloud located at a distance of $\approx 400 \mathrm{pc}\left(-10\right.$ to $\left.0 \mathrm{~km} \mathrm{~s}^{-1}\right)$ and measured in units of average temperature (from 0.75 to $1.5 \mathrm{~K}$ in steps of $0.25 \mathrm{~K}$ ).

within $15^{\circ}$ around the RoI were modeled to produce energy spectra and to calculate the test statistics (TS; Mattox et al. 1996) of the source. With the FST gtlike tool and an unbinned maximum likelihood fitting procedure, the energy spectrum of 2FGL J1507.0-622 has been derived. For this purpose, all sources listed in the Fermi-LAT 2-year catalogue in the RoI were modeled according to their best fit model. In order to guarantee stable fit results and since there are 65 identified Fermi-LAT sources in this region of the sky, only the flux at the decorrelation energy has been left as free parameter in the minimisation procedure. All other parameters have been fixed to their catalogue value. The Galactic and Extragalactic background components were modeled with the files gal_2yearp7v6_v0.fits and iso_p $7 v 6$ source.txt ${ }^{2}$, respectively. At the HESS J1507-622 position a TS value of 60 , corresponding to a statistical significance of $\sim 7.7 \sigma$ is found. The energy spectrum is best described by a power law in energy with $\Gamma=1.7 \pm 0.1_{\text {stat }} \pm 0.2_{\text {sys }}$ and integral flux between $(0.3-300) \mathrm{GeV}$ of $F=\left(2.0 \pm 0.5_{\text {stat }} \pm 1.0_{\text {sys }}\right) \times$ $10^{-9} \mathrm{~cm}^{-2} \mathrm{~s}^{-1}$. The flux in each energy band as shown in Figs. 2 and 3 has been calculated following the same procedure as described above.

The systematic error of the spectral result has been estimated by employing alternative source models, where other spectral parameters such as the spectral index for power-lawtype sources and/or the normalisation of 2FGL sources within $5^{\circ}$ of 2FGL J1507.0-622 have been left free in the minimisation procedure. As can be seen in Fig. 1, there is some residual emission above $10 \mathrm{GeV}$ energies, not covered by the Galactic diffuse model and partly coincident with a molecular cloud located at $\sim 400$ pc distance (Dame et al. 2001; Acero et al. 2011). Given the distance of 2FGL J1507.0-622 to the CO peak of $\sim 1^{\circ}$, no significant contribution to the 2FGL J1507.0-622 emission is expected. Indeed, including an additional point-like source at the

\footnotetext{
2 http://fermi.gsfc.nasa.gov/ssc/data/access/lat/ BackgroundModels.html
} 


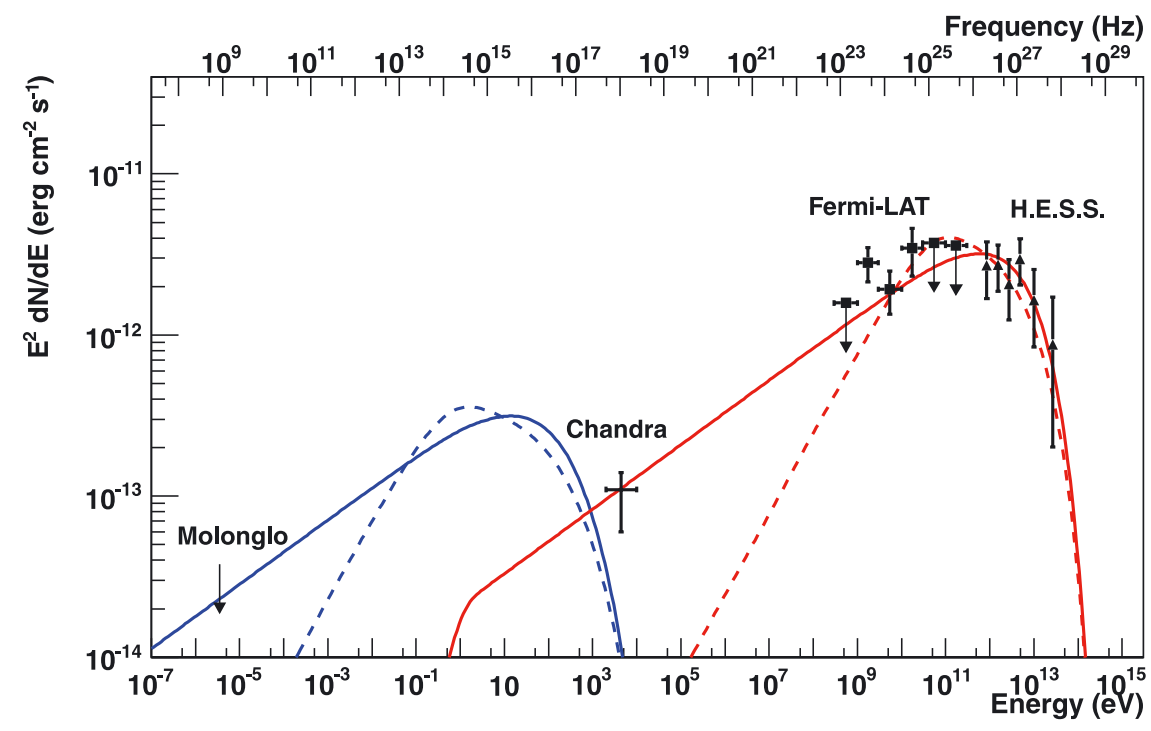

Fig. 2. Observed and calculated leptonic SED for HESS J1507-622 for a continuous injection of electrons. Two cases are shown: solid lines represent a model with an assumed (young) objects age of $10^{4}$ years and rather steep electron injection spectrum of $\Gamma_{\mathrm{e}}=2.6$; the dashed lines represent a rather old object with an age of $3 \times 10^{5}$ years and an electron injection spectrum with $\Gamma_{\mathrm{e}}=2.0$. For more details on the modeled SED see main text.

peak $\mathrm{CO}$ emission in the model did not change the fit results by more than $10 \%$ above $1 \mathrm{GeV}$ (20\% below $1 \mathrm{GeV})$.

\section{Spectral energy distribution}

The Fermi results presented in the previous section together with H.E.S.S. measurements are now used to study the SED of HESS J1507-622. Additionally, Acero et al. (2011) found diffuse X-ray emission using Chandra within the extend of HESS J1507-622 (magenta circle in Fig. 3 in Acero et al. 2011). This source features a flux of $\left(1.1_{-0.5}^{+0.3}\right) \times 10^{-13} \mathrm{erg} \mathrm{cm}^{-2} \mathrm{~s}^{-1}$ in the energy band of $(2-10) \mathrm{keV}$. This is the only extended X-ray source inside the VHE gamma-ray source and thus it is a potential counterpart to HESS J1507-622. However, it has to be noted that a firm association between the diffuse X-ray source and the VHE gamma-ray source can not be established (see Acero et al. 2011). To investigate possible constraints on the magnetic field, here it is assumed that this extended X-ray source is a counterpart to HESS J1507-622. Finally, the position of this source has also been covered by the second epoch Molonglo Galactic plane Survey in radio at $843 \mathrm{MHz}$ (Murphy et al. 2007). No source has been detected at the position of HESS J1507-622 within the sensitivity limit of this survey of $10 \mathrm{mJy} / \mathrm{beam}$ (beam size: $45 \times 45|\delta| \operatorname{arcsec})$. The extend of HESS J1507-622 contains about 450 beams. If the sensitivity per beam is integrated over the whole area of the gamma-ray source then the limit on the radio flux from HESS J1507-622 would be conservatively $4.5 \mathrm{Jy}$.

The observed SED does not strongly support a smooth power-law continuation with slope $\Gamma=2.24$ from HESS J1507622 to the HE regime (see Fig. 2) and is suggestive of spectral curvature. It is apparently compatible with a flat spectrum with $\Gamma \simeq 2$ but also a rising slope from the HE to the VHE gammaray energy range cannot be excluded. With the available data it is not possible to discriminate between a hadronic and a leptonic scenario for HESS J1507-622 based on the spectral form (see e.g. Figs. 2, 3) and hence both scenarios are discussed in the following.

\subsection{Leptonic model}

In Fig. 2, a computed SED for the simple case of a one zone model (see Hinton \& Aharonian 2007) is compared to the observational data. Given the fact that very little is known about HESS J1507-622, this simple approach was chosen in order to avoid too many implicit assumptions on the nature of the source. Due to the off-plane location of HESS J1507-622 it is furthermore reasonable to assume that only photons from the cosmicmicrowave background (CMB) are up-scattered to VHE energies by $\mathrm{HE}$ electrons and that contributions from other target radiation fields (e.g. starlight) can be neglected. In order not to overproduce synchrotron emission in the X-ray band in this scenario, the strength of the magnetic field was assumed to be $B=1 \mu \mathrm{G}$. As a first approach a quite young age of $10^{4}$ years of the source is assumed and electrons are continuously injected during this period. In this case the rather flat observed SED from the HE to the VHE gamma-ray energy range requires a rather steep spectral index of the injected electrons. Figure 2 demonstrates that such a leptonic scenario can in principal reproduce the observational data (input parameters: electron spectra following a power law with exponential cut-off with index $\Gamma_{\mathrm{e}}=2.6$ and cutoff energy $E_{\mathrm{c}, \mathrm{e}}=60 \mathrm{TeV}$, total energy in electrons $E_{\mathrm{e}}=2.5 \times$ $10^{49}(d / 1 \mathrm{kpc})^{2}$ erg, a magnetic field of $B=1 \mu \mathrm{G}$, and age of $10^{4}$ years). The constraints on the magnetic field strength inferred here differ from the value estimated in Acero et al. (2011) of $B=0.5 \mu \mathrm{G}$ due to the different spectral shape of the considered electron population $\left(\Gamma_{\mathrm{e}}=2.2\right.$ in Acero et al. 2011). This leptonic model would furthermore predict a radio flux density of about $2 \mathrm{Jy}$ at the Molonglo frequency of $843 \mathrm{MHz}$. That would be comparable to the radio limit on the entire area of the gamma-ray source. As a further constraint, this model would require a quite low density of the ambient medium of $\lesssim 0.1 \mathrm{~cm}^{-3}$. For higher densities bremsstrahlung would start to become dominant below a few $\mathrm{GeV}$ energies for such a steep electron spectrum. It has to be noted that, since the SED of HESS J1507-622 is observationally not very well constrained the parameters of this leptonic model are not unique to describe the observations (see e.g. Figs. 2, 3).

The population of electrons required to explain the gamma-ray emission in a leptonic scenario could in principle be generated by the spin-down energy loss of a pulsar. In the case where the pulsar is significantly older than its characteristic spin-down time the evolution of the population of electrons can be approximated by a burst like injection followed by IC and synchrotron cooling. For a review on the evolution 


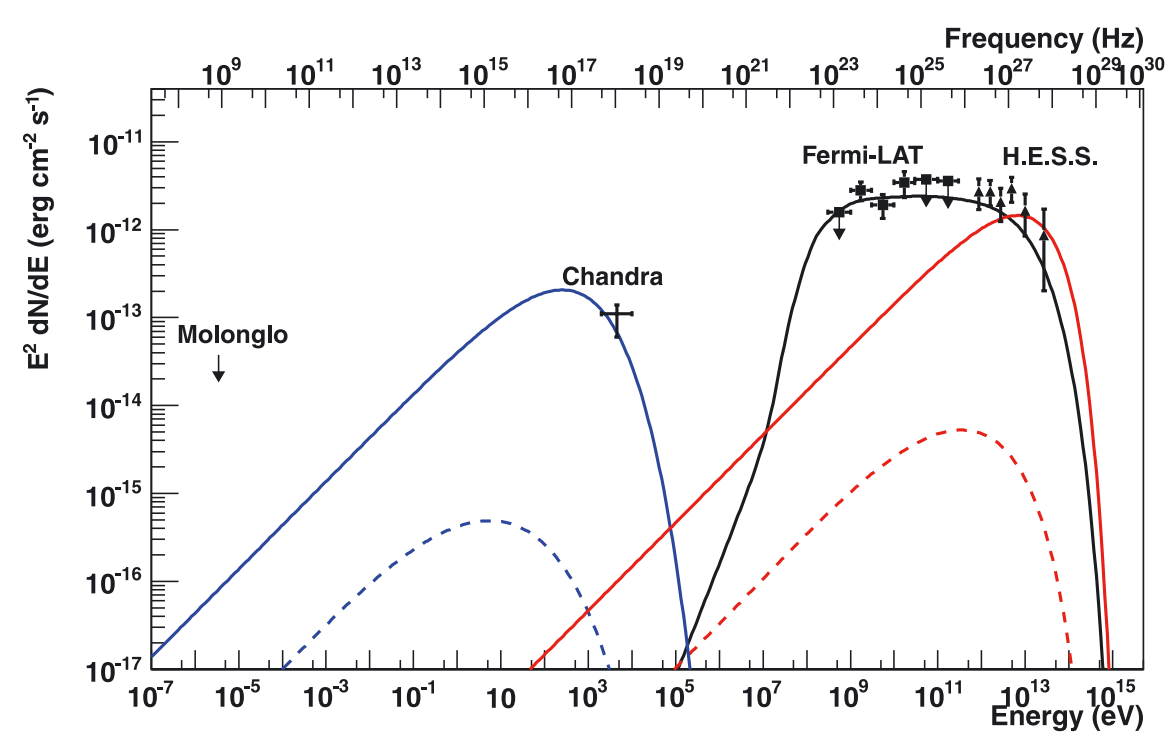

Fig. 3. Observed and calculated hadronic SED for HESS J1507-622 for a continuous injection of protons. In blue and red synchrotron and IC radiation of primary (solid lines) and secondary (dashed lines) electrons is shown for a source age of $10^{4}$ years. For more details see main text. of PWNe see Gaensler \& Slane (2006). Evolved PWNe with an age $\gg 10^{4}$ years can appear as rather bright VHE sources with only faint multi-wavelength counterparts (de Jager et al. 2009).

As a second possibility for a leptonic model that exhibits a flat gamma-ray spectrum from the HE to the VHE gamma-ray energy band a model is chosen where electrons with a hard spectral index are continuously injected over a quite long period of time and interact with target magnetic and radiation fields (Hinton \& Hofmann 2009) (input parameters: electron spectra following a power law with exponential cut-off with in$\operatorname{dex} \Gamma_{\mathrm{e}}=2.0$ and cut-off energy $E_{\mathrm{c}, \mathrm{e}}=60 \mathrm{TeV}$, total energy in electrons $E_{\mathrm{e}}=2 \times 10^{47}(d / 1 \mathrm{kpc})^{2}$ erg, a magnetic field of $B=1 \mu \mathrm{G})$. With these parameters it is found that an age of the source of $3 \times 10^{5}$ years would be required to explain the gamma-ray emission in the GeV range (see Fig. 2). Thus HESS J1507-622 could in principle also be explained by a rather old object containing a cooling population of electrons that is continuously injected.

\subsection{Hadronic model}

In Fig. 3, a computed hadronically dominated model is compared to the observed SED. For a comprehensive discussion of this hadronic model see Domainko (2011). The input parameters for this model are: total energy in hadronic cosmic rays $E_{\mathrm{CR}}=10^{50} \mathrm{erg}$, spectral index of injected protons of $\Gamma=2.0$, cutoff energy $E_{\mathrm{c}, \mathrm{CR}}=100 \mathrm{TeV}$, distance $d=2 \mathrm{kpc}$, density of the ambient medium $n_{\mathrm{H}}=1 \mathrm{~cm}^{-3}$, electron to proton ratio $\epsilon=5 \times 10^{-4}$, a magnetic field of $B=1 \mu \mathrm{G}$ and an age of $10^{4}$ years. The predicted radio flux density at $843 \mathrm{MHz}$ of this model is $5 \mathrm{mJy}$, compatible with the non-detection in the Molonglo survey. This scenario would require a rather young SNR and such a model has been regarded as an unlikely explanation for HESS J1507-622 (Domainko 2011). This conclusion is based on the compactness of the VHE source measured in Acero et al. (2011). As can be seen from Fig. 3 the IC and synchrotron contribution from secondary electrons for an object age of $10^{4}$ years is orders of magnitude below the IC and synchrotron emission from primary electrons. Note that only for object ages of $\gtrsim 10^{6}$ years emission from secondary electrons become important but even for this case they are still compatible with the radio limits and the Chandra flux point.
A second possibility for a hadronic scenario is given by the location on the sky of HESS J1507-622 in the direction of the outskirts of a molecular cloud (see Acero et al. 2011). In this scenario a locally enhanced cosmic ray density at the source location could result in detectable hadronic gamma-ray production. However, it has to be noted that in Acero et al. (2011) it has been concluded that the substantial difference in extension and an offset of $\sim 1^{\circ}$ of the VHE source centroid from the densest part of the molecular cloud suggests no obvious association between these two objects (see also Fig. 1). The molecular cloud is most likely located at a distance of about $400 \mathrm{pc}$. Therefore, a total energy in hadronic cosmic rays of $E_{\mathrm{CR}} \approx 2.4 \times$ $10^{47}\left(\mathrm{n} / 10 \mathrm{~cm}^{-3}\right)$ erg ( $n$ : density of target material) would be required to explain the gamma-ray emission. This population of cosmic rays would have to be concentrated in a sphere with radius of about $1 \mathrm{pc}$ (corresponding to an angular radius of $0.15^{\circ}$ at $400 \mathrm{pc}$ ). Consequently, the energy density in cosmic rays at HESS J1507-622 for an assumed density of target material of $n \approx 10 \mathrm{~cm}^{-3}$ would have to exceed the local cosmic ray density at the solar system by about three orders of magnitude. The absence of any plausible particle accelerator in the vicinity of HESS J1507-622 points against such a possibility and thus also this scenario may not provide a convincing explanation for the nature of the source.

In contrast to these hadronic scenarios here in the following a leptonic model for HESS J1507-622 is considered.

\section{Discussion}

In this section the implications of a leptonic model in general and an evolved PWN origin in particular for HESS J1507622 are discussed. This scenario can explain the appearance of the source as relatively bright VHE gamma-ray emitter with only faint low-energy counterpart (de Jager 2008; Mattana et al. 2009).

\subsection{Estimation of the distance}

The distance to an extended unidentified source can in principle be estimated if its physical size can be constrained. VHE particles escape from their production site by diffusion and for the simple case of point-like injection the size of a source is given by 
the diffusion coefficient and the age of the particles. For the case of old leptonic sources, the size of the object is limited by energy losses of the VHE electrons and thus the age of these particles is equivalent to the electron cooling time. At locations far away from the Galactic plane the dominating photon field responsible for energy losses of the VHE electrons is the CMB with an energy density of about $0.3 \mathrm{eV} \mathrm{cm}^{-3}$. For this case the cooling time of electrons is given by $t_{\text {cool }} \approx 10^{6}\left(E_{\mathrm{e}} / 1 \mathrm{TeV}\right)$ years (Hinton $\&$ Hofmann 2009) with $E_{\mathrm{e}}$ being the electron energy. The slowest possible diffusion is Bohm diffusion with a diffusion coefficient of $\kappa_{\mathrm{Bohm}} \approx 10^{26}\left(E_{\mathrm{e}, \mathrm{TeV}}\right) /\left(B_{\mu \mathrm{G}}\right) \mathrm{cm}^{2} \mathrm{~s}^{-1}$ where $E_{\mathrm{e}, \mathrm{TeV}}$ gives the electron energy in $\mathrm{TeV}$ and $B_{\mu \mathrm{G}}$ gives the magnetic field in $\mu \mathrm{G}$. With these assumptions the physical extension of a cooling dominated leptonic source can be estimated by $r \approx \sqrt{2 \kappa_{\mathrm{Bohm}} t_{\mathrm{cool}}}$. For electrons with an energy of $60 \mathrm{TeV}$ and a magnetic field of $1 \mu \mathrm{G}$ corresponding to $\kappa_{\mathrm{Bohm}} \approx 6 \times 10^{27} \mathrm{~cm}^{2} \mathrm{~s}^{-1}$ and $t_{\text {cool }} \approx$ $2 \times 10^{4}$ years the source would have a radius of about $25 \mathrm{pc}$. This estimate is also comparable to the typical sizes of evolved PWN which are abundant extended VHE gamma-ray sources in the Galactic plane (e.g. Mattana et al. 2009; Kargaltsev \& Pavlov 2010). If HESS J1507-622 has indeed an extension of 25 pc its angular radius of $0.15^{\circ}$ would imply a distance of about $10 \mathrm{kpc}$. It has to be noted that a larger magnetic field would result in slower diffusion and thus in a more compact source morphology. However, the low X-ray flux in comparison to the gamma-ray flux does not support such a possibility (see Fig. 2).

The more complete multi-wavelength picture of HESS J1507-622 might place further constraints on its distance. The SED fit from Sect. 3 seems to indicate a quite low magnetic field of $\sim 1 \mu \mathrm{G}$ which is significantly smaller than the typical Galactic magnetic field of $3-10 \mu \mathrm{G}$. One possibility for the source being situated in a region with low magnetic field is a location far below the plane and thus outside the magnetic field of the Galactic disk. Simard-Normandin \& Kronberg (1980) found a thickness of the magneto-ionic plane of the Milky Way of about $1.4 \mathrm{kpc}$. Thus, if HESS J1507-622 would be hosted by a low magnetic field environment outside this plane, its distance would exceed $20 \mathrm{kpc}$. Such a situation would imply then a physical radius of the object of $\gtrsim 50 \mathrm{pc}$. For comparison, this value corresponds roughly to the extension of the largest VHE gamma-ray emitting PWN found so far (Kargaltsev \& Pavlov 2010).

\subsection{Energetics of the source}

The flux of HESS J1507-622 corresponds to a luminosity of $L_{\gamma}(>1 \mathrm{TeV}) \approx 8 \times 10^{32}(d / 1 \mathrm{kpc})^{2} \operatorname{erg~s}^{-1}$ with $d$ being the distance to the source in kpc. For the distance estimates from Sect. 4.1 of $10 \mathrm{kpc}(20 \mathrm{kpc})$ this would result in a VHE gammaray luminosity of about $8 \times 10^{34} \mathrm{erg} \mathrm{s}^{-1}\left(3 \times 10^{35} \mathrm{erg} \mathrm{s}^{-1}\right)$ which is again comparable to the most luminous PWN detected so far (e.g. Mattana et al. 2009; Kargaltsev \& Pavlov 2010).

To estimate the total energy in electrons required to explain the luminosity of the gamma-ray source, two different approaches are considered here.

Firstly, only the population of electrons which can directly be probed by the gamma-ray observations are considered. As has been shown in Sect. 3 gamma-ray emission from HESS J1507622 has been detected down to the (1-3) GeV band which requires electrons with energies of about $100 \mathrm{GeV}$ for IC scattering on the CMB. These electrons IC cool on a timescale of $t_{\text {cool }} \approx 10^{7}$ years. Since the total flux in the Fermi energy range is $F_{\gamma} \approx 8.2 \times 10^{-12} \mathrm{erg} \mathrm{cm}^{-2} \mathrm{~s}^{-1}$ the total energy in electrons above $100 \mathrm{GeV}$, given by $E_{\text {tot }} \approx F_{\gamma} t_{\text {cool }} 4 \pi d^{2}$, amounts to $3 \times$ $10^{47}(d / 1 \mathrm{kpc})^{2}$ erg with $d$ being the distance to the source.

Secondly, for determining the total energy in electrons the entire energy range for the electrons from the sub-relativistic regime up to the cutoff at $60 \mathrm{TeV}$ is considered. It is further assumed that the electron distribution over the whole energy range can be described by a power-law distribution with index 2.6. From the calculated SED it is found that a total energy in electrons for this case of about $2.5 \times 10^{49}(d / 1 \mathrm{kpc})^{2} \mathrm{erg}$ would be needed to explain the luminosity of the source.

With the estimates from the previous paragraphs the energetics in electrons for the putative source distances can be determined. A distance of $10 \mathrm{kpc}(20 \mathrm{kpc})$ would require a total energy in VHE electrons of about $3 \times 10^{49} \mathrm{erg}\left(1.2 \times 10^{51} \mathrm{erg}\right)$ for the case where only the gamma-ray producing electrons are considered or $2.5 \times 10^{50} \mathrm{erg}\left(10^{52} \mathrm{erg}\right)$ for the case where the entire energy range for the electrons is taken into account. These energy requirements would be compatible with the typical spindown energy of a pulsar of a few times $10^{49}$ erg (Gaensler \& Slane 2006) for the first case up to a distance of about $10 \mathrm{kpc}$ but would exceed the typical energetics of pulsar wind nebulae for the second case. For the second case a pulsar can only provide the necessary energy in electrons if the source is located within a distance of a few kpc. The compact morphology of HESS J1507-622 seems to challenge this scenario (see Sect. 4.1).

\subsection{PWN interpretation and potential Galactic halo location}

The rather compact appearance of HESS J1507-622 favours a multi-kpc distance $d$ of the source (see Sect. 4.1) which in turn results in a considerable physical offset from the Galactic plane of about $600(d / 10 \mathrm{kpc})$ pc. Such a location has certain implications for a PWN scenario for the VHE gamma-ray source since pulsars are generally born in the Galactic disk. However, pulsars can be ejected from their birth place due to natal kicks that results in a Galactic lateral distribution for young pulsars which follows a Gaussian distribution with age dependent scale height. The width of this distribution scales linearly with pulsar age and for pulsar ages $\lesssim 1 \mathrm{Myr}$ it is expected to be $\lesssim 500$ pc (Sun \& Han 2004). Consequently, gamma-ray PWNe hosting pulsars with ages typically shorter than a few times $10^{5}$ years are expected to be found at offsets considerably smaller than $500 \mathrm{pc}$ and thus at smaller offsets than has been estimated for HESS J1507622. Even for extreme cases of pulsar velocities of $1000 \mathrm{~km} \mathrm{~s}^{-1}$ (Hobbs et al. 2005) the pulsar would need $6 \times 10^{5} \mathrm{yr}$ to travel 600 pc. A pulsar located at such a large distance from the plane would be older than the typical gamma-ray PWN hosting pulsar and its age would have exceeded the IC cooling time of electrons that are energetic enough to produce VHE gamma-rays (see Sect. 4.1). Thus, the off-plane location of HESS J1507-622 challenges a PWN origin for this source.

This limitation on a PWN origin can in principle be overcome by a scenario where the pulsar is born offset from the Galactic disk in the core-collapse of a massive star that was ejected from the Galaxy (see Zinn et al. 2011, for core-collapse supernovae connected to hypervelocity stars). Since the presupernova lifetime of massive stars is of the order of $10^{7}$ years, significantly longer than the lifetime of young energetic pulsars, such a star can reach larger offsets from the Galactic plane. Consequently, after the star has exploded as a supernova, an energetic pulsar could be found at such an off-plane location. For this scenario it would be expected that a SNR shell should be found in the neighbourhood of the PWN. Therefore 
one restriction for such a model is the absence of a SNR shell in the vicinity of HESS J1507-622. Gamma-ray PWNe could be much older than the duration of the Sedov phase of the SNR. In this case the very old PWN would be very extended. Deep future VHE gamma-ray observations that investigate the presence of emission with lower surface brightness on larger spatial scales could be used to test this scenario.

It has further to be noted that supernovae of hypervelocity stars appear to be rare events (Zinn et al. 2011). Therefore only a very limited number of PWNe resulting from such supernovae are expected in the Milky Way. Consequently, this scenario could also be tested by surveys that search for similar sources as HESS J1507-622 (see Sect. 4.4).

The possibility that HESS J1507-622 belongs to the Galactic halo discussed in this section is based on the observational result that the source is rather compact and located at significant angular distance from the Galactic plane. However, future more sensitive VHE gamma-ray observations could in principle reveal that the source is more extended than measured to date. In this case, the constraints on the distance and on the physical offset from the Galactic plane would be relaxed and a PWN interpretation would appear more feasible. A decreased distance to HESS J1507-622 would also result in a decreased gammaray luminosity and hence also a less powerful pulsar would be required to explain the VHE gamma-ray source.

To summarise, the properties of HESS J1507-622 challenge a PWN origin but due to the uncertainty in distance such a scenario cannot be ruled out in general.

\subsection{Potential population of such objects}

In this section an alternative to a PWN scenario is explored. The following considerations are based on the result that with HESS J1507-622 and 2FGL J1507.0-6223 an unassociated HE source has been linked to an unassociated VHE source (Nolan et al. 2012). It is further based on the discovery of a considerable number of unassociated HE sources reported in the Fermi 2-year catalogue (Nolan et al. 2012). In principle some of these HE sources could feature extended VHE emission similar to HESS J1507-622.

So far only one VHE gamma-ray source without prominent multi-wavelength counterpart, namely HESS J1507-622 has been found outside the main distribution of such sources within $\pm 2^{\circ}$ around the Galactic plane (Chaves et al. 2009). However, HESS J1507-622 could in principle be the first discovered representative of a source population which is distributed with large angular offset around the Galactic disk. In this case the distribution of this object population can be used to further constrain the distance and thus nature of their representatives. For the simplest case, where sources from this population are distributed homogeneously around the Earth, the surface density of these objects should be constant in every direction. Since the distance to the source is likely to be several kpc (see Sect. 4.1) it may belong to a population of objects which is distributed around the Galactic centre rather than to a local source population around the Earth. In this case the surface number density should be more concentrated around the Galactic centre.

HESS J1507-622 is a rather bright VHE source and is observed with about $8 \%$ of the flux of the Crab nebula (Acero et al. 2011). Therefore, the H.E.S.S. Galactic plane survey would have sufficient sensitivity for detecting a source like HESS J1507622 out to a Galactic latitude of $\pm 4^{\circ}$ and the result that only one such source has been found can be used to constrain their number on the sky. Since the H.E.S.S. Galactic plane survey extends over $140^{\circ}$ in Galactic longitude the surface density of objects like HESS J1507-622 in the bands of $-4^{\circ}$ to $-2^{\circ}$ and $2^{\circ}$ to $4^{\circ}$ Galactic latitude should not be much larger than one source per $\sim 0.17$ steradians. For an order of magnitude estimate on the number of comparable sources here a constant source number density is assumed as would be expected for a local source population. In this case $\$ 70$ sources would be distributed on the entire sky. For different distributions (e.g. concentrated on the Galactic centre as discussed above) this estimate is only a course guideline. Nevertheless, in principle $O(10)$ similar sources might be scattered on the sky. Surveys obtained with the future Cherenkov Telescope Array (CTA; Actis et al. 2011) and HAWC $^{3}$ will be crucial to test if HESS J1507-622 belongs to a population of sources with similar characteristics.

\section{Summary and outlook}

In this paper the SED from $\mathrm{GeV}$ to $\mathrm{TeV}$ energies for HESS J1507-622 has been explored and additional results from radio and $\mathrm{X}$-ray observations from the literature have been used to further constrain the emission scenarios. Based on the spectral results and with the available data it is not possible to discriminate between a hadronic and a leptonic scenario. The location and compactness of the source may indicate a considerable physical offset from the Galactic disk for this object. Interestingly, such a scenario would imply that this source is disconnected from a young stellar population. So far only very few Galactic VHE gamma-ray sources that are related to an old stellar population have been found. Such sources include remnants of supernovae type Ia (Acero et al. 2010; Acciari et al. 2011) and most likely a globular cluster (Abramowski et al. 2011b), but none of them lacks a prominent low-energy emitter in its angular vicinity. The nature of HESS J1507-622 is still unknown to date, but a PWN scenario cannot be ruled out in general. More sensitive VHE gamma-ray observations are desirable to search for spatially more extended, low surface brightness VHE gamma-ray emission from this source. At the same time, further MWL observations in the X-ray and radio band appear to be necessary to constrain the nature of the gamma-ray emitter. These observations may also help to explore if this source is indeed linked to an old stellar population.

HESS J1507-622 could be the first representative of a class of VHE gamma-ray sources with HE counterpart that are distributed with larger physical offset around the Galactic disk. In this context it is important to note that several hundred HE gamma-ray sources have been reported in the Fermi 2-year catalogue (Nolan et al. 2012) that appear to be unassociated with counterparts in other wavelength. In principle some of them could be linked to extended VHE gamma-ray sources similar to HESS J1507-622. Future surveys in the VHE gamma-ray range are necessary to explore whether there is indeed a Galactic halo source population in this energy range.

Acknowledgements. S.O. acknowledges the support of the Humboldt foundation by a Feodor-Lynen fellowship. The authors want to thank V. Marandon for providing the CO map and J. A. Hinton for valuable discussion. The authors acknowledge support from their host institutions.

\section{References}

Abdo, A. A., Ackermann, M., Ajello, M., et al. (Fermi LAT collaboration) 2011, ApJ, 734, 28

Abramowski, A., Acero, F., Aharonian, F., et al. (H.E.S.S. collaboration) 2011a, A\&A, 531, A81

${ }^{3}$ http://hawc.umd.edu/ 
W. Domainko and S. Ohm: Exploring the nature of HESS J1507-622

Abramowski, A., Acero, F., Aharonian, F., et al. (H.E.S.S. collaboration) 2011b, A\&A, 531, L18

Acciari, V. A., Aliu, E., Arlen, T., et al. (VERITAS collaboration) 2011, ApJ, 730, L20

Acero, F., Aharonian, F., Akhperjanian, A. G., et al. (H.E.S.S. collaboration) 2010, A\&A, 516, A62

Acero, F., Aharonian, F., Akhperjanian, A. G., et al. (H.E.S.S. collaboration) 2011, A\&A, 525, A45

Actis, M., Agnetta, G., Aharonian, F., et al. (CTA collaboration) 2011, Exp. Astron., 32, 193

Aharonian, F., Akhperjanian, A. G., Aye, K.-M., et al. (H.E.S.S. collaboration) 2005, Science, 307, 1938

Aharonian, F., Akhperjanian, A. G., Barres de Almeida, U., et al. (H.E.S.S collaboration ) 2008, A\&A, 477, 353

Atwood, W. B., Abdo, A. A., Ackermann, M., et al. (Fermi-LAT collaboration) 2009, ApJ, 697, 1071

Chaves, R. C. G. for the H.E.S.S. collaboration 2009 [arXiv: 0907. 0768]

Dame, T. M., Hartmann, D., \& Thaddeus, P. 2001, ApJ, 547, 792

Domainko, W. F. 2011, AdSpR, 47, 640

Gaensler, B. M., \& Slane, P. O. 2006, ARA\&A, 44, 17

Hinton, J. A., \& Aharonian, F. A. 2007, ApJ, 657, 302

Hinton, J. A., \& Hofmann, W. 2009, ARA\&A, 47, 523
Hobbs, G., Lorimer, D. R., Lyne, A. G. \& Kramer, M. 2005, MNRAS, 360, 974 de Jager, O. C. 2008, ApJ, 678, L113

de Jager, O. C., Ferreira, S. E. S., Djannati-Ataï, A., et al. 2009, Proc. 31st Int. Cosm. Ray Conf., Lodz, Poland, July [arXiv: 0906.2644]

Kargaltsev, O., \& Pavlov, G. G. 2010, AIPC, 1248, 25

Landi, R., de Rosa, A., Dean, A. J., et al. 2007, MNRAS, 380, 929

Mattana, F., Falanga, M., Götz, D., et al. 2009, ApJ, 694, 12

Mattox, J. R., Bertsch, D. L., Chiang, J., et al. 1996, ApJ, 461, 396

Murphy, T., Mauch, T., Green, A., et al. 2007, MNRAS, 382, 382

Nolan, P. L., Abdo, A. A., Ackermann, M., et al. (Fermi LAT collaboration) 2012, ApJS, 199, 31

Simard-Normandin, M., \& Kronberg, P. P. 1980, ApJ, 242, 74

Sun, X. H., \& Han, J. L. 2004, MNRAS, 350, 232

Tam, P. H. T., Wagner, S. J., Tibolla, O., \& Chaves, R. C. G. 2010, A\&A, 518, A8

Tian, W. W., Leahy, D. A., Haverkorn, M., \& Jiang, B. 2008, ApJ, 679, L85

Tibolla, O., Mannheim, K., Kaufmann, S., \& Elsässer, D. 2011, Proc. of the 32nd ICRC, Beijing, China [arXiv: 1109.3144]

Torres, D. F., Li, H., Chen, Y., et al. 2011, MNRAS, 417, 3072

Yamazaki, R., Kohri, K., Bamba, A., et al. 2006, MNRAS, 371, 1975

Zinn, P.-C., Grunden, P., \& Bomans, D. J. 2011, A\&A, 536, A103 